\title{
Tailoring the absorption bandwidth of graphene at critical coupling
}

\author{
Shuyuan Xiao $\odot,{ }^{1,2, *}$ Tingting Liu $\odot,{ }^{3, \dagger}$ Xing Wang, ${ }^{1}$ Xiaojun Liu, ${ }^{1,2}$ and Chaobiao Zhou ${ }^{4}$ \\ ${ }^{1}$ Institute for Advanced Study, Nanchang University, Nanchang 330031, China \\ ${ }^{2}$ Jiangxi Key Laboratory for Microscale Interdisciplinary Study, Nanchang University, Nanchang 330031, China \\ ${ }^{3}$ School of Physics and Electronics Information, Hubei University of Education, Wuhan 430205, China \\ ${ }^{4}$ College of Mechanical and Electronic Engineering, Guizhou Minzu University, Guiyang 550025, China
}

(Received 21 May 2020; revised 17 July 2020; accepted 21 July 2020; published 7 August 2020)

\begin{abstract}
The ability to manipulate the absorption bandwidth has enabled a wide range of applications, from narrowband lasing emission to broadband photodetection. Applying the concept to emerging two-dimensional materials has the potential to enable breakthroughs in advanced compact photonic and optoelectronic devices. Here we present a general method for tailoring the absorption bandwidth of graphene via critical coupling in the near-infrared regime. In a simple two-port resonant structure, dissipative graphene is integrated with a lossless photonic crystal slab, achieving a significant bandwidth manipulation up to 100 times from ultra-narrowband $(<0.50 \mathrm{~nm})$ to broadband $(>50 \mathrm{~nm})$ with a maximum absorption efficiency of 0.5 . The modulation mechanism lies in the intrinsic dependence of bandwidth on the relationship between radiation rate and dissipative loss rate, which is fulfilled by changing the structure parameters and graphene conductivity. This work offers an effective route to engineer light-graphene interactions and shows great prospects in designing high-performance graphene-based devices with enhanced efficiency and flexibility.
\end{abstract}

DOI: 10.1103/PhysRevB.102.085410

\section{INTRODUCTION}

Since its successful exfoliation in 2004, two-dimensional graphene has attracted broad interest owing to its extraordinary electrical and optical properties, spurring the rapid exploration and development in novel photonic and optoelectronic devices [1]. In the visible and near-infrared range, a major challenge of graphene-based devices is that light absorption of undoped monolayer graphene can be as low as $2.3 \%$ at normal incidence stemming from the atomic-scale thickness [2,3], which severely hinders the quantum efficiency and photoresponsivity. On this issue, hybrid configurations with resonant structures offer myriad strategies to engineer light-graphene interactions associated with resonance frequency, absorption efficiency, and bandwidth in a flexible way. Rooted in this understanding, different methods have been proposed within the regime by integrating graphene with plasmonic nanoparticles [4,5], Fabry-Pérot microcavities [6,7], metal gratings [8,9], one- [10], two- $[11,12]$, and three-dimensional photonic crystals [13], metamaterials and metasurfaces [14,15], showing the feasibility of various applications, including modulators, photodetectors, and lasing devices.

\footnotetext{
*syxiao@ncu.edu.cn

${ }^{\dagger}$ ttliu@hue.edu.cn
}

Published by the American Physical Society under the terms of the Creative Commons Attribution 4.0 International license. Further distribution of this work must maintain attribution to the author(s) and the published article's title, journal citation, and DOI.
Recently, the concept of critical coupling has been explored to tailor light-graphene interactions in the visible and near infrared. The incident light can be maximally absorbed when the radiation rate of energy out of the resonator equals the dissipative loss rate in the configuration, which is called the critical coupling condition. Piper and Fan theoretically proposed the total absorption of monolayer graphene via critical coupling to a guided resonance at optical frequencies and then experimentally demonstrated it $[16,17]$. Afterwards, different resonant structures were designed to boost the absorption efficiency in graphene via the critical coupling condition, and this mechanism also inspired the exploration of absorption engineering in the whole family of two-dimensional materials [18-28]. On the other hand, in sharp contrast to extensive studies on absorption efficiency, little research has been devoted to the absorption bandwidth in the critical coupling system. Given the fact that the absorption bandwidth plays a vital role in practical application scenarios, including lasing emission [29], color filtering, switching [30,31], light modulation $[32,33]$, photodetection, and energy harvesting [34,35], it is clearly of great interest to investigate the bandwidth manipulation of graphene in such systems.

In this work, we propose a general method to tailor the absorption bandwidth of graphene via critical coupling in the near infrared, using a simple two-port resonant structure composed of a graphene-covered two-dimensional photonic crystal slab. Based on the coupled-mode theory (CMT), the radiation rate and the dissipative loss rate in the system jointly determine the absorption efficiency and bandwidth. By adjusting the values of these two rates by tuning structure parameters and graphene conductivity, we have achieved bandwidth manipulation from ultra-narrowband $(<0.50 \mathrm{~nm})$ 
to broadband $(>50 \mathrm{~nm}$ ). This method demonstrates the large modulation of absorption bandwidth while maintaining the efficiency at a theoretical maximum value, which suggests advantages in efficiency and flexibility of high-performance graphene-based emitters, detectors, modulators, filters, and so on.

\section{REVIEW OF COUPLED-MODE THEORY}

Without the loss of generality, we consider the input-output behavior of a mirror-symmetric resonator with a single-mode resonance coupled to two identical ports. Assuming there is no dissipative loss in the system, the optical behavior can be described by CMT equations [36-39],

$$
\begin{gathered}
\frac{d a}{d t}=\left(i \omega_{0}-\gamma\right) a+D^{T}\left|s_{+}\right\rangle, \\
\left|s_{-}\right\rangle=C\left|s_{+}\right\rangle+D a,
\end{gathered}
$$

where $a$ is the resonance amplitude of the mode, with $|a|^{2}$ corresponding to the energy stored in the resonator, $\omega_{0}$ and $\gamma$ represent the resonance frequency and radiation rate of the mode, respectively, $\left|s_{+}\right\rangle=\left[s_{1+}, s_{2+}\right]^{T}$ represents the input amplitude of plane waves, with $\left|s_{+}\right|^{2}$ equal to the input power and a similar term for the output, $\left|s_{-}\right\rangle=\left[s_{1-}, s_{2-}\right]^{T}, C$ represents a $2 \times 2$ background scattering matrix with reflection and transmission between the ports in the absence of resonance, and $D$ gives the coupling constants between the resonance mode and the ports in the form $D=\left[d_{1}, d_{2}\right]^{T}$. The coefficients are related by energy conservation and time-reversal symmetry constraints, and the output amplitude $\left|s_{-}\right\rangle$can be directly described using the scattering matrix $S$ of the system for externally incident $\left|s_{+}\right\rangle$,

$$
\left|s_{-}\right\rangle=S\left|s_{+}\right\rangle=\left[C+\frac{D D^{T}}{i\left(\omega-\omega_{0}\right)+\gamma}\right]\left|s_{+}\right\rangle .
$$

For this two-port system with mirror symmetry, the background scattering matrix takes a special form,

$$
C=\exp (i \phi)\left[\begin{array}{ll}
r_{d} & i t_{d} \\
i t_{d} & r_{d}
\end{array}\right],
$$

where $r_{d}$ and $t_{d}$ are the direct reflection and transmission coefficients, with $r_{d}^{2}+t_{d}^{2}=1$, and $\phi$ is a real constant. Consequently, the scattering matrix $S$ of the system can be deduced as

$$
\begin{aligned}
S= & \exp (i \phi)\left\{\left[\begin{array}{cc}
r_{d} & i t_{d} \\
i t_{d} & r_{d}
\end{array}\right]\right. \\
& \left.+\frac{\gamma}{i\left(\omega-\omega_{0}\right)+\gamma}\left[\begin{array}{ll}
-\left(r_{d} \pm i t_{d}\right) & \mp\left(r_{d} \pm i t_{d}\right) \\
\mp\left(r_{d} \pm i t_{d}\right) & -\left(r_{d} \pm i t_{d}\right)
\end{array}\right]\right\} .
\end{aligned}
$$

Here the \pm sign represents the even and odd resonance modes. The intensity reflection coefficient $R=\left|S_{11}\right|^{2}$ and transmission coefficient $T=\left|S_{21}\right|^{2}$ are derived as

$$
\begin{aligned}
& R=\frac{r_{d}^{2}\left(\omega-\omega_{0}\right)^{2}+t_{d}^{2} \gamma^{2} \mp 2 r_{d} t_{d}\left(\omega-\omega_{0}\right) \gamma}{\left(\omega-\omega_{0}\right)^{2}+\gamma^{2}}, \\
& T=\frac{t_{d}^{2}\left(\omega-\omega_{0}\right)^{2}+r_{d}^{2} \gamma^{2} \pm 2 r_{d} t_{d}\left(\omega-\omega_{0}\right) \gamma}{\left(\omega-\omega_{0}\right)^{2}+\gamma^{2}} .
\end{aligned}
$$

Further, we consider the case where the input is incident from only a single port, with $D D^{T}=2 \gamma$ and a general time dependence of $a=a_{0} e^{i \omega t}$, the energy stored in the resonator can be derived from Eqs. (1) and (2),

$$
|a|^{2}=\frac{\gamma}{\left(\omega-\omega_{0}\right)^{2}+\gamma^{2}} .
$$

When a dissipative loss is introduced into such a system and does not affect the underlying symmetry of the structure, Eq. (1) can be modified as $d a / d t=\left(i \omega_{0}-\gamma-\delta\right) a+$ $D^{T}\left|s_{+}\right\rangle$, where $\delta$ is the dissipative loss rate of the system. Then the stored energy $|a|^{2}$ can be updated by replacing the term $\gamma^{2}$ in the denominator with $(\gamma+\delta)^{2}$. As a result, absorption of the system finally becomes

$$
A=\frac{2 \delta \gamma}{\left(\omega-\omega_{0}\right)^{2}+(\gamma+\delta)^{2}} .
$$

From the theoretical expressions above, the light absorption performance of the system is determined by the radiation loss and the dissipative loss. At the resonance frequency which determines the position of the absorption spectrum, i.e., $\omega=$ $\omega_{0}$, the absorption is determined by the ratio between $\gamma$ and $\delta$,

$$
A_{0}=\frac{2}{\frac{\gamma}{\delta}+\frac{\delta}{\gamma}+2},
$$

with a maximum value of $A_{0}=0.5$ when the radiation rate is equal to the dissipative loss rate $\gamma=\delta$, satisfying the critical coupling condition.

In such a system, the transmission and reflection spectrum exhibits an asymmetric Fano line shape, while the absorption spectrum shows a symmetric Lorentzian line shape. In general, the absorption bandwidth is defined as the full width at half maximum (FWHM). If the half-maximum absorption $A_{1}$ occurs at frequency $\omega_{1}, \Gamma^{\mathrm{FWHM}}=2\left|\omega_{1}-\omega_{0}\right|$ can be deduced from Eq. (9),

$$
\Gamma^{\mathrm{FWHM}}=2(\gamma+\delta)
$$

Consequently, the bandwidth of the absorption spectrum is determined by the sum of the radiation rate and loss rate.

\section{STRUCTURE DESIGN AND NUMERICAL MODEL}

The proposed absorption structure is a two-port system composed of a monolayer graphene deposited on top of a twodimensional photonic crystal slab, as illustrated in Fig. 1. The photonic crystal slab is made from a sheet of silicon consisting of a periodic array of cylindrical air holes on a square grid, which is characterized by lattice period $p=800 \mathrm{~nm}$ in both the $x$ and $y$ directions, thickness $h=100 \mathrm{~nm}$, and air hole radius initially being set at $r=125 \mathrm{~nm}$. For material modeling, silicon is selected as the building block owing to its low-loss feature with a refractive index of $n=3.5$, and monolayer graphene serves as an ultrathin lossy film with controllable optical surface conductivity. The lossless photonic crystal slab underneath supports guided resonance with electromagnetic energy strongly confined within the slab, acting as the leaky resonator.

The surface conductivity of graphene can be derived by the random-phase approximation in the local limit, including 


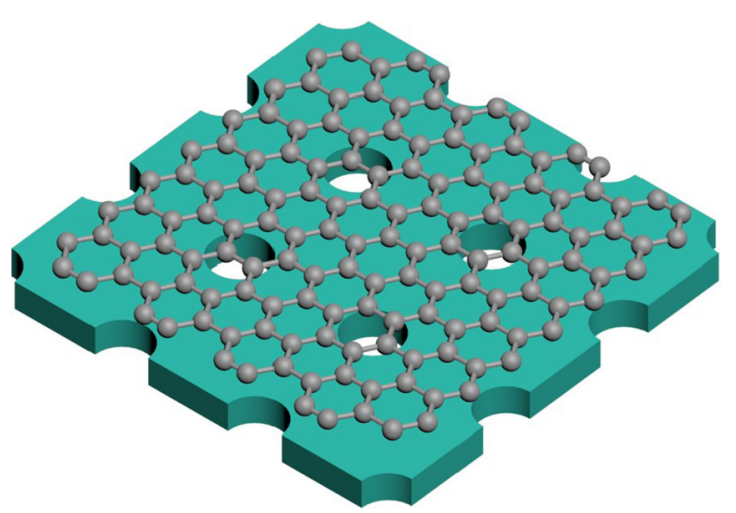

FIG. 1. Schematic of a two-port system with a graphene layer deposited on a two-dimensional photonic crystal slab.

intraband and interband transitions [40-43],

$$
\begin{aligned}
\sigma_{\mathrm{g}}= & \frac{2 e^{2} k_{\mathrm{B}} T}{\pi \hbar^{2}} \frac{i}{\omega+i \tau^{-1}} \ln \left[2 \cosh \left(\frac{E_{\mathrm{F}}}{2 k_{\mathrm{B}} T}\right)\right] \\
& +\frac{e^{2}}{4 \hbar}\left[\frac{1}{2}+\frac{1}{\pi} \arctan \left(\frac{\hbar \omega-2 E_{\mathrm{F}}}{2 k_{\mathrm{B}} T}\right)\right. \\
& \left.-\frac{i}{2 \pi} \ln \frac{\left(\hbar \omega+2 E_{\mathrm{F}}\right)^{2}}{\left(\hbar \omega-2 E_{\mathrm{F}}\right)^{2}+4\left(k_{\mathrm{B}} T\right)^{2}}\right],
\end{aligned}
$$

where $e, k_{B}, T, \hbar$, and $\omega$ are the electron charge, the Boltzmann constant, the environment temperature, the reduced Planck's constant, and the incident light frequency, respectively. $\tau$ is the carrier relaxation time and depends on the carrier mobility $\mu$, the Fermi level $E_{\mathrm{F}}$, and the Fermi velocity $v_{\mathrm{F}}$ with the relationship $\tau=\left(\mu E_{\mathrm{F}}\right) /\left(e v_{\mathrm{F}}^{2}\right)$, where $\mu=10000 \mathrm{~cm}^{2} / \mathrm{V} \mathrm{s}$ and $v_{\mathrm{F}}=1 \times 10^{6} \mathrm{~m} / \mathrm{s}$ are adopted here. According to Eq. (12), the variations of the graphene conductivity as a function of incident wavelength and Fermi level are displayed in Fig. 2. The real part of the graphene conductivity dramatically decreases once the Fermi level increases from the Dirac point by half of the photon energy, i.e., $E_{\mathrm{F}}>\hbar \omega / 2$, because the interband transition is Pauli blocked, leading to the rapid reducing of graphene dissipative loss. This variation process can be captured by the steplike behavior in Fig. 2(a). In contrast, the imaginary part shows a continuous increase due to the relatively significant intraband transition as the Fermi level increases in Fig. 2(b). Therefore, the surface conductivity of graphene is subject to the Fermi level $E_{\mathrm{F}}$, offering the possibility to flexibly tailor the absorption performance of the system.
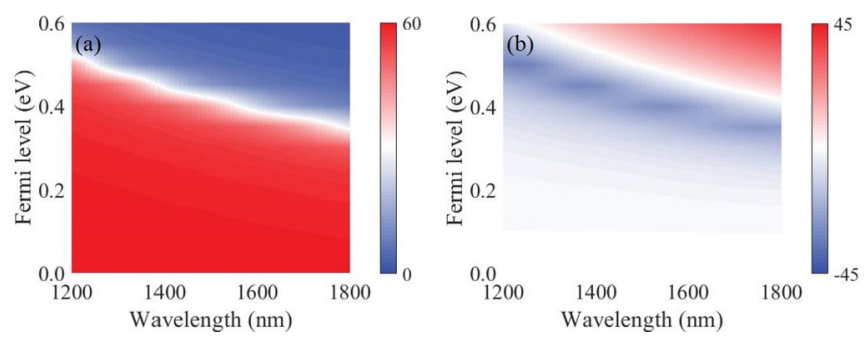

FIG. 2. (a) Real part and (b) imaginary part of graphene conductivity as a function of wavelength and Fermi level.
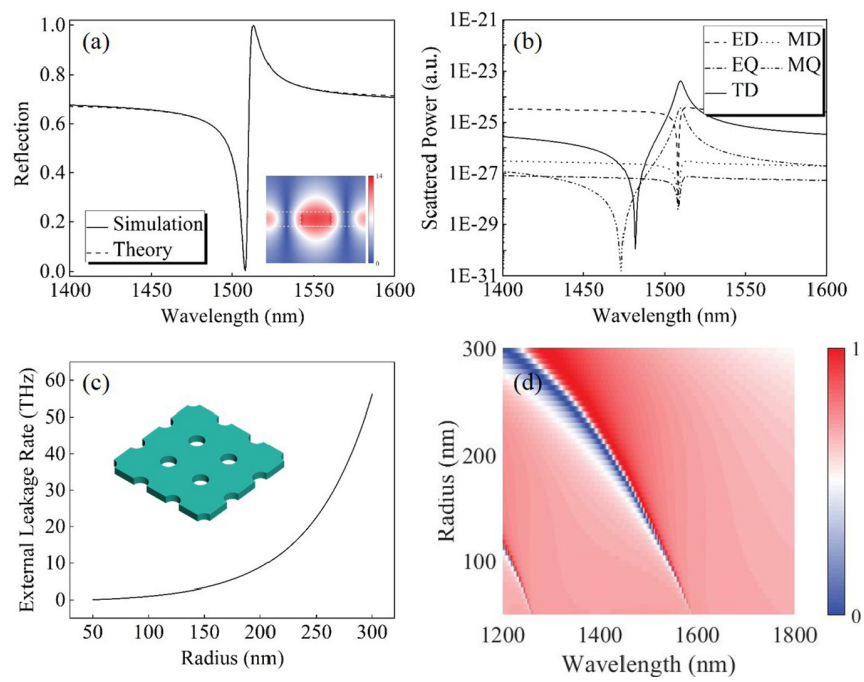

FIG. 3. (a) Simulated and theoretical reflection spectra and the corresponding electric field distribution of $\left|E_{y z}\right|$ on the $y$ - $z$ crosssectional plane through the center of the air hole for the bare photonic crystal slab. (b) Contributions of multipole moments, including the electric dipole (ED), magnetic dipole (MD), electric quadrupole (EQ), magnetic quadrupole (MQ), and toroidal dipole (TD) to the far-field radiation of the guided resonance under the Cartesian coordinates. (c) The radiation rate $\gamma$ of the photonic crystal slab as a function of air hole radius. (d) The reflection spectra of the bare photonic crystal slab as a function of incident wavelength and air hole radius.

The numerical simulations are performed with the finitedifference time-domain method. In the calculations, a moderate mesh grid is adopted to make a good trade-off between accuracy, memory requirements, and simulation time. The plane wave is incident along the $-z$ direction, periodic boundary conditions are utilized in the $x$ and $y$ directions, and perfectly matched layer absorbing boundaries are adopted in the $z$ direction.

\section{TAILORING THE ABSORPTION BANDWIDTH OF GRAPHENE}

\section{A. Dependence of the radiation rate on structure parameters}

For a comprehensive understanding of the functionality of the guided resonance in the structure, the bare silicon photonic crystal slabs without the top graphene layer are first studied under normal illumination. It has long been known that the guided mode in photonic crystal slabs interacts with external radiation under phase-matched coupling, generating the leaky guided resonance [44-46]. As Fig. 3(a) shows, the guided resonance in the bare slab refers to Fano resonance with an asymmetric line shape, a quasibound state arising from the coupling of an in-plane discrete guided mode, and continuum free-space radiation modes $[47,48]$, which are contributed from multipole moments and can be decomposed under the Cartesian coordinates in Fig. 3(b). According to Eq. (6), the theoretical fitting curve of the reflection spectrum based on CMT matches the simulated result well, and the electric field distribution reveals that it is the lowest even mode. The radiation rate of the guided resonance can be obtained as 
$\gamma=1.95 \mathrm{THz}$, while the dissipative loss rate is zero due to the lossless silicon slab here.

Moreover, the external radiation rate $\gamma$ can be controlled by the filling fraction $r / p$ associated with the air hole radius and the period, providing the possibility to tune the optical response through critical coupling. Here, as the air hole radius $r$ increases from 50 to $300 \mathrm{~nm}$ with the fixed period $p=$ $800 \mathrm{~nm}$, the radiation rate $\gamma$ increases in Fig. 3(c) because of the increasing scattering of the guided resonance into the free space. As a direct consequence, the simulated reflection spectra exhibit increasing bandwidth across the wavelength regime of interest, as shown in Fig. 3(d). On the other hand, the increasing radius $r$ also adjusts the resonance wavelength to the lower wavelength regions owing to the reducing effective refractive index of the structure. In principle, the other parameters, such as period $p$, thickness $h$, and the refractive index $n$, can also be considered to control $\gamma$. It can be inferred that $\gamma$ can be adjusted over a wide range by changing these structure parameters.

\section{B. Narrowing the absorption bandwidth of graphene}

When monolayer graphene is integrated into the photonic crystal slab, it behaves as the only absorption material in the proposed structure and determines the dissipative loss rate $\delta$ of this two-port system. By simply tuning graphene conductivity, the dissipative loss rate $\delta$ can be varied. As mentioned in Sec. III, one of the most promising characteristics of graphene is the dynamically tunable optical surface conductivity associated with the Fermi level via applying bias voltage, chemical doping, or optical stimulus. Hence, the absorption bandwidth of graphene can be manipulated by adjusting the dissipative rate $\delta$ by shifting the Fermi level while maintaining critical coupling by simultaneously varying the radiation rate $\gamma$. In more detail, $\delta$ is supposed to be reduced by decreasing graphene conductivity with increasing the Fermi level as in Fig. 2, while $\gamma$ is simultaneously reduced by decreasing the air hole radius as in Fig. 3.

Figure 4 illustrates the absorption spectrum of undoped and doped monolayer graphene with respect to the air hole radius and incident wavelength. As shown in Fig. 4(a), when the undoped graphene (with Fermi level $E_{\mathrm{F}}=0 \mathrm{eV}$ ) is integrated in the structure, the critical coupling point can be found by modulation in the two parameters, and the maximum absorption efficiency of 0.5 is realized for an air hole radius of 125 nm. In Fig. 4(b), the absorption bandwidth of the structure reaches $9.52 \mathrm{~nm}$. From Eqs. (9) and (11), the theoretical values of the radiation rate and the dissipative rate are fitted as $\gamma=\delta=1.95 \mathrm{THz}$, and the corresponding bandwidth is 9.5 $\mathrm{nm}$, which matches well the simulated result. When graphene is doped, the Pauli state-blocking effect would become significant around $0.4 \mathrm{eV}$ as the Fermi level of graphene further increases, specifically, the blocking point of the Fermi level ranges from 0.52 to $0.34 \mathrm{eV}$, corresponding to the wavelength regime from 1200 to $1800 \mathrm{~nm}$, which will remarkably reduce the dissipative loss. Here doped graphene with Fermi levels of 0.4 and $0.5 \mathrm{eV}$ is taken to show the tunable narrowband absorption in Figs. 4(c) and 4(e). In these two cases, the critical coupling occurs by choosing the proper air hole radius of $r=110 \mathrm{~nm}$ and $r=57.5 \mathrm{~nm}$, respectively. As shown in
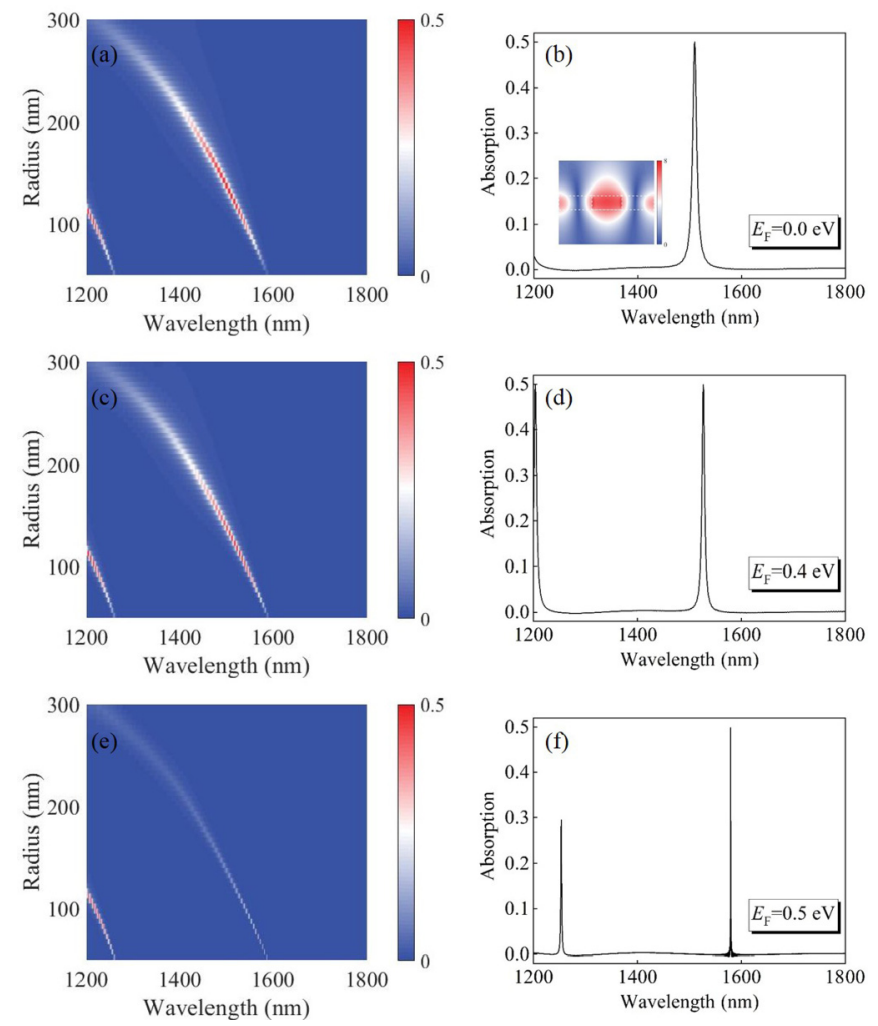

FIG. 4. Realization of narrowband absorption of graphene via shifting the Fermi level. (a), (c), and (e) show the absorption spectra as a function of the incident wavelength and air hole radius, and (b), (d), and (f) provide the simulated absorption spectra at critical coupling for monolayer graphene with different Fermi levels of $E_{\mathrm{F}}=0,0.4,0.5 \mathrm{eV}$ in the proposed structure.

Figs. 4(d) and 4(f), the absorption bandwidth of graphene can be narrowed to 5.94 and $0.47 \mathrm{~nm}$. These results indicate that the narrowband or even ultra-narrowband absorption of monolayer graphene in the two-port system can be achieved by simply shifting the Fermi level of graphene at critical coupling, in turn verifying the bandwidth control from the relationship between the dissipative loss and radiation rate of the system.

\section{Broadening the absorption bandwidth of graphene}

The discussions above demonstrate that the absorption bandwidth of graphene can be narrowed in a simple way. On the other hand, how to freely broaden the bandwidth is another important issue in practical applications, such as photodetection. According to the tuning strategy, it is expected that the absorption bandwidth may be broadened by simultaneous increases of the dissipative loss rate $\delta$ and radiation rate $\gamma$ while maintaining the critical coupling condition. It is interesting that the stacked graphene layers act as isolated monolayer graphene due to the electrical decoupling, and herein dissipative loss of graphene shows a linear increase with the layer number [2,3]. Based on this fact, the increase of $\delta$ could be achieved by increasing the layer number of graphene. At the same time, the increase of the other parameter, $\gamma$, can also be realized by increasing the air hole radius. 

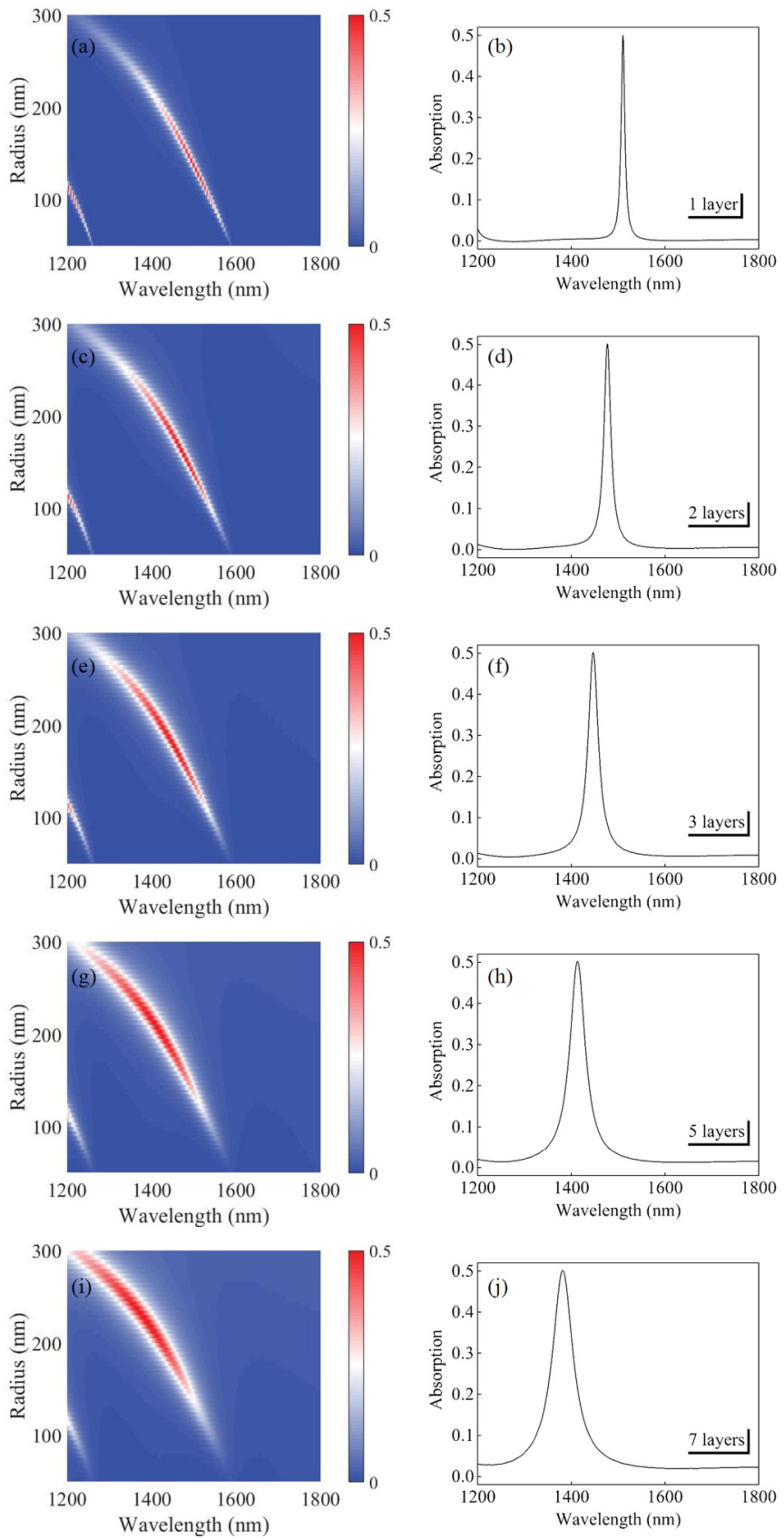

FIG. 5. Realization of broadband absorption of graphene by changing the layer number. (a), (c), (e), (g), and (i) show the absorption spectra as a function of incident wavelength and air hole radius, and (b), (d), (f), (h), and (j) provide the simulated absorption spectra at critical coupling for graphene with different layer numbers of $N=1,2,3,5$, and 7 in the proposed structure.

In order to investigate the broadband absorption by increasing the layer number of graphene, the absorption spectrum as a function of wavelength and air hole radius is investigated and presented in Fig. 5. In the numerical modeling, the rest of the parameters of the system remain the same as those in Fig. 4. The layer number of graphene is set to 1, 2, 3, 5, and 7 with the Fermi level fixed as $E_{\mathrm{F}}=0 \mathrm{eV}$. It can be seen that the bandwidth of graphene absorption continuously increases as the graphene layer number increases due to the larger dissipative loss rate $\delta$. Similarly, the critical coupling points with a maximum absorption efficiency of 0.5 can be easily found by sweeping the air hole radius $r$. For example, the absorption bandwidth of the graphene layer at critical coupling is $17.69,27.16,41.67$, and $56.7 \mathrm{~nm}$ for a layer number of 2, 3, 5, and 7 with $r=155,180,205$, and $225 \mathrm{~nm}$, respectively. Therefore, the absorption bandwidth of graphene can be tuned over a wide range in a properly crafted structure, which essentially provides a promising route for broadband light-harvesting devices.

\section{CONCLUSIONS}

In summary, a general method has been proposed to tailor the absorption bandwidth of graphene at critical coupling in the near-infrared regime. In the proposed simple twoport structure, graphene as a dissipative material is incorporated with a lossless photonic crystal slab, achieving manipulation from ultra-narrowband $(<0.50 \mathrm{~nm})$ to broadband $(>50 \mathrm{~nm})$ at maximum absorption. The physical mechanism of the tunable absorption bandwidth is based on the intrinsic dependence of the bandwidth on the relationship between the radiation rate and dissipative loss rate, i.e., $\Gamma^{\mathrm{FWHM}}=2(\gamma+$ $\delta$ ) at critical coupling condition $\gamma=\delta$, which is fulfilled by changing the structure parameter of the photonic crystal slab and surface conductivity of the graphene layer. By varying the Fermi level of graphene and the air hole radius, the narrowband and even ultra-narrowband can be attained over a bandwidth range from 9.52 to $0.47 \mathrm{~nm}$. Likewise, broadband absorption at critical coupling is also demonstrated with a modulation regime from 9.52 to $56.7 \mathrm{~nm}$ by increasing the layer number of graphene. The impressive modulation of absorption bandwidth in graphene provides a great opportunity to engineer light-graphene interaction in a simple and robust way and holds great potential for graphene-based optoelectronic devices, including narrowband light emitters, color filters, and switching, as well as broadband photodetectors and energy harvesting devices. Furthermore, our proposed critical coupling system can also be utilized for active optical devices, such as light spatial modulators, by simply shifting the Fermi level of graphene. Finally, the method of tailoring absorption bandwidth is general, is not limited to graphene, and can be applied to other lossy two-dimensional materials.

\section{ACKNOWLEDGMENTS}

This work is supported by the National Natural Science Foundation of China (Grants No. 61775064, No. 11847132, No. 11947065, and No. 61901164), the Natural Science Foundation of Jiangxi Province (Grant No. 20202BAB211007), the Interdisciplinary Innovation Fund of Nanchang University (Grant No. 2019-9166-27060003), the Natural Science Research Project of Guizhou Minzu University (Grant No. GZMU[2019]YB22), and the China Scholarship Council (Grant No. 202008420045). The authors would also like to thank Dr. S. Li for her guidance on the effective multipole expansion and Dr. X. Jiang for beneficial discussions on the critical coupling mechanism. 
[1] F. Bonaccorso, Z. Sun, T. Hasan, and A. C. Ferrari, Nat. Photonics 4, 611 (2010).

[2] R. R. Nair, P. Blake, A. N. Grigorenko, K. S. Novoselov, T. J. Booth, T. Stauber, N. M. R. Peres, and A. K. Geim, Science 320, 1308 (2008).

[3] K. F. Mak, M. Y. Sfeir, Y. Wu, C. H. Lui, J. A. Misewich, and T. F. Heinz, Phys. Rev. Lett. 101, 196405 (2008).

[4] Y. Liu, R. Cheng, L. Liao, H. Zhou, J. Bai, G. Liu, L. Liu, Y. Huang, and X. Duan, Nat. Commun. 2, 579 (2011).

[5] Z. Fang, Z. Liu, Y. Wang, P. M. Ajayan, P. Nordlander, and N. J. Halas, Nano Lett. 12, 3808 (2012).

[6] M. Furchi, A. Urich, A. Pospischil, G. Lilley, K. Unterrainer, H. Detz, P. Klang, A. M. Andrews, W. Schrenk, G. Strasser, and T. Mueller, Nano Lett. 12, 2773 (2012).

[7] A. Ferreira, N. M. R. Peres, R. M. Ribeiro, and T. Stauber, Phys. Rev. B 85, 115438 (2012).

[8] B. Zhao, J. M. Zhao, and Z. M. Zhang, Appl. Phys. Lett. 105, 031905 (2014).

[9] B. Zhao, J. M. Zhao, and Z. M. Zhang, J. Opt. Soc. Am. B 32, 1176 (2015).

[10] J.-T. Liu, N.-H. Liu, J. Li, X. J. Li, and J.-H. Huang, Appl. Phys. Lett. 101, 052104 (2012).

[11] W. Wang, A. Klots, Y. Yang, W. Li, I. I. Kravchenko, D. P. Briggs, K. I. Bolotin, and J. Valentine, Appl. Phys. Lett. 106, 181104 (2015).

[12] C. Zhou, G. Liu, G. Ban, S. Li, Q. Huang, J. Xia, Y. Wang, and M. Zhan, Appl. Phys. Lett. 112, 101904 (2018).

[13] X. Zhang and S. John, Phys. Rev. B 99, 035417 (2019).

[14] S. Song, Q. Chen, L. Jin, and F. Sun, Nanoscale 5, 9615 (2013).

[15] Y. Cai, J. Zhu, and Q. H. Liu, Appl. Phys. Lett. 106, 043105 (2015).

[16] J. R. Piper and S. Fan, ACS Photonics 1, 347 (2014).

[17] Y. Liu, A. Chadha, D. Zhao, J. R. Piper, Y. Jia, Y. Shuai, L. Menon, H. Yang, Z. Ma, S. Fan, F. Xia, and W. Zhou, Appl. Phys. Lett. 105, 181105 (2014).

[18] H. Lu, B. P. Cumming, and M. Gu, Opt. Lett. 40, 3647 (2015).

[19] C.-C. Guo, Z.-H. Zhu, X.-D. Yuan, W.-M. Ye, K. Liu, J.-F. Zhang, W. Xu, and S.-Q. Qin, Adv. Opt. Mater. 4, 1955 (2016).

[20] H. Li, M. Qin, L. Wang, X. Zhai, R. Ren, and J. Hu, Opt. Express 25, 31612 (2017).

[21] X. Jiang, T. Wang, S. Xiao, X. Yan, L. Cheng, and Q. Zhong, Nanotechnology 29, 335205 (2018).

[22] A. Akhavan, S. Abdolhosseini, H. Ghafoorifard, and H. Habibiyan, J. Lightwave Technol. 36, 5593 (2018).

[23] Y. M. Qing, H. F. Ma, and T. J. Cui, Opt. Express 26, 32442 (2018).

[24] S. Xiao, T. Liu, L. Cheng, C. Zhou, X. Jiang, Z. Li, and C. Xu, J. Lightwave Technol. 37, 3290 (2019).

[25] J. Wang, A. Chen, Y. Zhang, J. Zeng, Y. Zhang, X. Liu, L. Shi, and J. Zi, Phys. Rev. B 100, 075407 (2019).
[26] T. Liu, X. Jiang, C. Zhou, and S. Xiao, Opt. Express 27, 27618 (2019).

[27] T. Liu, X. Jiang, H. Wang, Y. Liu, C. Zhou, and S. Xiao, Appl. Phys. Express 13, 012010 (2020).

[28] Z.-Q. Cheng, X. Luo, L. Xu, X. Zhai, and L.-L. Wang, Opt. Express 28, 14151 (2020).

[29] J. Wang, D. Han, A. Chen, Y. Dai, M. Zhou, X. Hu, Z. Yu, X. Liu, L. Shi, and J. Zi, Phys. Rev. B 96, 195419 (2017).

[30] C. Valagiannopoulos, IEEE Antennas Propag. Mag. 57, 318 (2015).

[31] A. N. Papadimopoulos, N. V. Kantartzis, N. L. Tsitsas, and C. A. Valagiannopoulos, Appl. Opt. 56, 9779 (2017).

[32] P. Cencillo-Abad, N. I. Zheludev, and E. Plum, Sci. Rep. 6, 37109 (2016).

[33] Y. Xia, J. Wang, Y. Zhang, Y. Shan, Y. Dai, A. Chen, T. Shen, S. Wu, X. Liu, L. Shi, and J. Zi, Adv. Opt. Mater., 2000264 (2020), doi: 10.1002/adom.202000264.

[34] M. H. Elshorbagy, E. López-Fraguas, J. M. Sánchez-Pena, B. García-Cámara, and R. Vergaz, Sol. Energy 202, 10 (2020).

[35] J. Li, X. Chen, Z. Yi, H. Yang, Y. Tang, Y. Yi, W. Yao, J. Wang, and Y. Yi, Mater. Today Energy 16, 100390 (2020).

[36] S. Fan, W. Suh, and J. D. Joannopoulos, J. Opt. Soc. Am. A 20 , 569 (2003).

[37] J. D. H. Morales, B. M. Rodríguez-Lara, and B. A. Malomed, Opt. Lett. 42, 4402 (2017).

[38] Y. Zhiyenbayev, Y. Kominis, C. Valagiannopoulos, V. Kovanis, and A. Bountis, Phys. Rev. A 100, 043834 (2019).

[39] Y. Ra'di, A. Krasnok, and A. Alù, ACS Photonics 7, 1468 (2020).

[40] L. A. Falkovsky and S. S. Pershoguba, Phys. Rev. B 76, 153410 (2007).

[41] J. Zhang, Z. Zhu, W. Liu, X. Yuan, and S. Qin, Nanoscale 7, 13530 (2015).

[42] S. Xiao, T. Wang, Y. Liu, C. Xu, X. Han, and X. Yan, Phys. Chem. Chem. Phys. 18, 26661 (2016).

[43] A. Sánchez-Arellano, J. Pérez-Huerta, D. Ariza-Flores, I. Sustaita-Torres, and J. Madrigal-Melchor, Superlattices Microstruct. 130, 68 (2019).

[44] S. Fan and J. D. Joannopoulos, Phys. Rev. B 65, 235112 (2002).

[45] A. E. Miroshnichenko, S. Flach, and Y. S. Kivshar, Rev. Mod. Phys. 82, 2257 (2010).

[46] C. Zhou, S. Li, Y. Wang, and M. Zhan, Phys. Rev. B 100, 195306 (2019).

[47] C. W. Hsu, B. Zhen, J. Lee, S.-L. Chua, S. G. Johnson, J. D. Joannopoulos, and M. Soljačić, Nature (London) 499, 188 (2013).

[48] A. S. Kupriianov, Y. Xu, A. Sayanskiy, V. Dmitriev, Y. S. Kivshar, and V. R. Tuz, Phys. Rev. Appl. 12, 014024 (2019). 